\title{
Assessing the utility of confirmatory studies following identification of large-scale genomic imbalances by microarray
}

\author{
Jennifer N. Sanmann, PhD ${ }^{1,2}$, Diane L. Pickering, MS'1, Denae M. Golden, BS', \\ Jadd M. Stevens, BS, BA ${ }^{1}$, Thomas E. Hempel, BS ${ }^{1}$, Pamela A. Althof, MS'1, Michele L. Wiggins, BA ${ }^{1}$, \\ Lois J. Starr, MD ${ }^{2,3}$, Bhavana J. Davé, $\mathrm{PhD}^{1,2}$ and Warren G. Sanger, PhD ${ }^{1-3}$
}

\begin{abstract}
Purpose: The identification of clinically relevant genomic dosage anomalies assists in accurate diagnosis, prognosis, and medical management of affected individuals. Technological advancements within the field, such as the advent of microarray, have markedly increased the resolution of detection; however, clinical laboratories have maintained conventional techniques for confirmation of genomic imbalances identified by microarray to ensure diagnostic accuracy. In recent years the utility of this confirmatory testing of large-scale aberrations has been questioned but has not been scientifically addressed.
\end{abstract}

Methods: We retrospectively reviewed 519 laboratory cases with genomic imbalances meeting reportable criteria by microarray and subsequently confirmed with a second technology, primarily fluorescence in situ hybridization.
Results: All genomic imbalances meeting reportable criteria detected by microarray were confirmed with a second technology. Microarray analysis generated no false-positive results.

Conclusion: Confirmatory testing of large-scale genomic imbalances (deletion of $\geq 150 \mathrm{~kb}$, duplication of $\geq 500 \mathrm{~kb}$ ) solely for the purpose of microarray verification may be unwarranted. In some cases, however, adjunct testing is necessary to overcome limitations inherent to microarray. A recommended clinical strategy for adjunct testing following identified genomic imbalances using microarray is detailed.

Genet Med advance online publication 15 January 2015

Key Words: confirmatory testing; copy-number variation; fluorescence in situ hybridization; genomic imbalances; microarray

\section{INTRODUCTION}

The identification of genomic imbalances that result in the loss or gain of clinically significant genetic material assists clinicians in the diagnosis, prognosis, and medical management of affected individuals. The tools available to assess genomic imbalances have advanced greatly in the past few decades, evolving from lower-resolution techniques such as conventional karyotyping and fluorescence in situ hybridization (FISH) to much higherresolution techniques such as single-nucleotide polymorphism microarray. ${ }^{1}$ Because of its detection ability and clinical utility, the guidelines set forth by the American College of Medical Genetics and Genomics currently recommend microarray as a first-tier test for patients with intellectual disability, autism, and multiple congenital anomalies. ${ }^{2,3}$

Historically, FISH has been considered a gold standard for confirmation of copy-number changes identified by microarray. ${ }^{4}$ However, high-density microarray platforms are identifying an increasing number of aberrations that cannot be confirmed using FISH, for a variety of reasons: (i) the aberration is smaller than the detection limits of the FISH technology, (ii) the aberration is located in a region of the genome for which a clone is not readily available, or (iii) the aberration is not a result of a genomic imbalance (i.e., a region of homozygosity). With the increasing familiarity of microarray analysis, the use of FISH or another secondary technology for confirmation of suspicious or pathogenic genomic imbalances identified by microarray has declined in some laboratories. However, others continue to verify the vast majority of genomic imbalances identified by their microarray platforms with FISH or another technology. The current literature lacks evidence to support or to refute the utility of routine confirmation of genomic imbalances identified by microarray in the clinical laboratory setting. In this study we retrospectively reviewed all adjunct studies performed for the purpose of confirming a genomic imbalance identified by microarray. The utility of the adjunct testing was assessed based on its ability to detect genomic imbalances erroneously identified by microarray (i.e., false positives).

\section{MATERIALS AND METHODS}

\section{Subjects}

In this retrospective study we included 519 consecutive cases with genomic dosage imbalance detected by microarray studies

\footnotetext{
${ }^{1}$ Human Genetics Laboratory, Munroe-Meyer Institute for Genetics and Rehabilitation, University of Nebraska Medical Center, Omaha, Nebraska, USA; ${ }^{2}$ Department of Pediatrics, University of Nebraska Medical Center, Omaha, Nebraska, USA; ${ }^{3}$ Department of Genetic Medicine, Munroe-Meyer Institute for Genetics and Rehabilitation, University of Nebraska Medical Center, Omaha, Nebraska, USA. Correspondence: Jennifer N. Sanmann (jsanmann@unmc.edu)
} 
(first-tier test) that were confirmed by another test. These cases were received in the Human Genetics Laboratory at the University of Nebraska Medical Center for diagnostic testing between May 2005 and December 2013. The cases selected for this review met the following inclusion criteria: (i) one or more genomic imbalances meeting reportable criteria were identified by microarray and (ii) adjunct testing was performed to confirm the presence of the genomic imbalance(s). All cases meeting the aforementioned inclusion criteria were included in the study regardless of the microarray platform (bacterial artificial chromosome; oligonucleotide; single-nucleotide polymorphism).

\section{DNA extraction}

Genomic DNA was isolated from these specimens using the Qiagen Blood and Cell Culture DNA Mini Kit or the Qiagen QIAamp DNA Blood Mini Kit (Qiagen, Valencia, CA). DNA quantity was determined using the NanoDrop ND-1000 spectrophotometer (NanoDrop Technologies, Wilmington, DE), and DNA quality was assessed by agarose gel electrophoresis.

\section{Microarray}

Microarray studies for the detection of genomic dosage anomalies were performed using a bacterial artificial chromosome, an oligonucleotide, or a single-nucleotide polymorphism microarray platform according to manufacturer instructions (Table 1 ), and the studies were analyzed following recommended guidelines. ${ }^{5-9}$ Genomic imbalances meeting reportable criteria were flagged for confirmatory studies.

\section{Fluorescence in situ hybridization}

FISH studies were performed on interphase and/or metaphase nuclei using commercially available or custom ("homebrew") FISH probes designed to target the aberrant region of interest in conjunction with a control locus. Before hybridization, slides were pretreated using an automated VP 2000 processor (Abbott Molecular, Des Plaines, IL) with standard protocols. Following pretreatment, the cells and probes were codenatured at 75-78 ${ }^{\circ} \mathrm{C}$ for $1-3 \mathrm{~min}$ and incubated overnight at $37-39^{\circ} \mathrm{C}$ using the HyBrite or ThermoBrite system (Abbott Molecular). Nuclei were counterstained with 4,6-diamidino-2-phenylindole in Antifade solution (Abbott Molecular), and the slides were analyzed using Olympus BX51 and Leica DM6000B fluorescence microscopes. Images were acquired and archived using the CytoVision Image Analysis System (Leica Microsystems, Buffalo Grove, IL).

\section{Multiplex ligation-dependent probe amplification}

Multiplex ligation-dependent probe amplification (MLPA) was performed per the manufacturer's instructions using kits specific for MECP2 and SHOX (P015-C and P018-F1, respectively) obtained from MRC-Holland (Amsterdam, The Netherlands). The polymerase chain reaction products were resolved on the ABI 3730 or the ABI 3500 capillary electrophoresis apparatus in conjunction with the GeneScan 500 ROX or the GeneScan 600 LIZ Size Standard (Applied Biosystems, Foster City, CA). Results were analyzed using the Peak Scanner Software or the Stand Alone Coffalyser Software (MRC-Holland), and result ratios were interpreted using the manufacturer's recommendations.

\section{Conventional cytogenetics}

Standard G-band analysis was performed on 72-h stimulated (phytohemagglutinin-M) suspension cultures from peripheral blood specimens. Images were acquired and archived using the CytoVision Image Analysis System (Leica Microsystems). Nomenclature was reported according to the International System for Human Cytogenetic Nomenclature (2013) guidelines. ${ }^{10}$

\section{RESULTS}

A total of 519 cases with genomic dosage imbalances and concurrent confirmatory testing performed between May 2005 and December 2013 at the Human Genetics Laboratory were reviewed. From the oligonucleotide and single-nucleotide polymorphism platforms, the smallest deletion was an intragenic aberration of $1.3-8.2 \mathrm{~kb}$, and the largest deletion, omitting whole-chromosome loss (e.g., Turner syndrome), was 55.9 megabases $(\mathrm{Mb})$. The observed duplications ranged in size from $137.1 \mathrm{~kb}$ to $62.0 \mathrm{Mb}$, omitting whole-chromosome

Table 1 Microarray platforms and resolutions

\begin{tabular}{|c|c|c|c|c|}
\hline \multicolumn{3}{|c|}{ Platform details } & \multicolumn{2}{|c|}{ Array resolution and reporting cutoff } \\
\hline Date & Type & Name & Deletions (kb) & Duplications (kb) \\
\hline May 2005-April 2007 & BAC & $\begin{array}{l}\text { 404-600 clones: Spectral Genomics } \\
\text { (Houston, TX) }\end{array}$ & Target regions & Target regions \\
\hline May 2005-April 2007 & BAC & 2,600 (1 Mb): Spectral Genomics & Whole genome: $\geq 1 \mathrm{Mb}$ & Whole genome: $\geq 1 \mathrm{Mb}$ \\
\hline May 2007-September 2010 & Oligonucleotide & $\begin{array}{l}\text { 44,000 (44K): Agilent Technologies, } \\
\text { Custom Design }{ }^{5} \text { (Santa Clara, CA) }\end{array}$ & $\begin{array}{l}\text { Target regions: } \geq 20-50 \mathrm{~kb} \text {; } \\
\text { whole genome: } \geq 250 \mathrm{~kb}\end{array}$ & $\begin{array}{l}\text { Target regions: } \geq 20-50 \mathrm{~kb} \text {; } \\
\text { whole genome: } \geq 250 \mathrm{~kb}\end{array}$ \\
\hline October 2010-January 2013 & Oligonucleotide & $\begin{array}{l}\text { 180,000 (180K): Agilent } \\
\text { Technologies, Custom Design }\end{array}$ & $\begin{array}{l}\text { Target regions: } \geq 5-10 \mathrm{~kb} \text {; } \\
\text { whole genome: } \geq 50 \mathrm{~kb}\end{array}$ & $\begin{array}{l}\text { Target regions: } \geq 5-10 \mathrm{~kb} \text {; } \\
\text { whole genome: } \geq 50 \mathrm{~kb}\end{array}$ \\
\hline February 2013-December 2013 & SNP & $\begin{array}{l}1.9 \text { million copy-number markers } \\
\text { and } 750,000 \text { genotypable SNPs: } \\
\text { Affymetrix Cytoscan HD (Santa } \\
\text { Clara, CA) }\end{array}$ & Whole genome: $>10 \mathrm{~kb}$ & Whole genome: $>10 \mathrm{~kb}$ \\
\hline
\end{tabular}

BAC, bacterial artificial chromosome; kb, kilobase; Mb, megabase; SNP, single-nucleotide polymorphism. 
gains. Aberration size was not calculated for the bacterial artificial chromosome arrays because of the low density and targeted nature of the platform. Of the 519 cases, 86 cases ( 17\%) exhibited two or more genomic imbalances, although not all were amenable to FISH (Table 2). From this cohort, all cases with genomic imbalances meeting reportable criteria that were identified by microarray analysis were verified with a second technology (FISH, $n=510$; an alternative microarray platform, $n=6$; multiplex ligation-dependent probe amplification, $n=2$; or conventional cytogenetics, $n=1$ ). Given that microarray results were confirmed by alternative testing in all cases, no false-positive results were generated by microarray analysis.

FISH studies definitively confirmed the reportable genomic imbalances identified by microarray in 98.3\% (510/519) of cases (Supplementary Figure S1 online). FISH was unable to conclusively verify 10 reportable genomic imbalances (5 deletions and 5 duplications) identified by microarray in a total of 9 cases. Of note, all identified discrepancies between the microarray and FISH technologies were a result of the limitations of the FISH probes used for confirmation. Specifically, seven copynumber variations were below the lower limits of detection by FISH (150 and $500 \mathrm{~kb}$ for deletions and duplications, respectively). Two additional genomic imbalances lacked overlap between the precise linear locations of the identified aberration and the FISH probes. In only one case the FISH probe used to confirm the microarray finding was entirely contained within the identified 747-kb duplication. Tandem duplication of very small regions may result in a lack of segregated signals, and, in this case, the FISH result was indeterminate because three distinct signals could not be consistently enumerated in the interphase cells analyzed. In all cases in which FISH confirmation was not achieved $(n=9)$, the genomic imbalances identified by microarray were confirmed with an alternate technology.

\section{DISCUSSION}

Across microarray platforms, the results of this retrospective analysis of 519 cases demonstrate that there were no falsepositive genomic dosage imbalances that met the reportable threshold using microarray analysis. In addition, the data show that the lack of concordance between the reportable genomic imbalances identified by microarray and FISH, one of the most time- and cost-efficient technologies available, originated exclusively from the limitations of the FISH assay. Thus, these data suggest that confirmation of larger-scale genomic

Table 2 Characterization of the genomic imbalances detected using microarray

\begin{tabular}{lc} 
Aberration type & Cases, $\boldsymbol{n}(\%)$ \\
\hline Sole deletion & $260(51)$ \\
Sole duplication & $164(32)$ \\
Deletion + duplication & $53(10)$ \\
Deletion + deletion & $16(3)$ \\
Duplication + duplication & $9(2)$ \\
$\geq 3$ deletions and/or duplications & $8(2)$
\end{tabular}

imbalances (deletions $\geq 150 \mathrm{~kb}$ and duplications $\geq 500 \mathrm{~kb}$ ) identified by microarray analysis with a second technology such as FISH solely for the purpose of microarray verification may be of limited use. These data support the most recent guidelines from the American College of Medical Genetics and Genomics, which do not include a recommendation to confirm all reportable genomic imbalances identified by microarray with a second technology in routine cases. ${ }^{9}$

In an era of increasingly dismal reimbursement for medical services, it is critical that laboratories contain operational costs. This cost containment, however, must not come at the expense of high-quality patient care. The results of this study suggest that a reduction in the number of performed confirmatory studies may provide an opportunity to minimize diagnostic testing costs without negatively impacting patient care. However, it is critical to acknowledge that this study does not diminish the utility of adjunct testing such as FISH following microarray for clinically necessary purposes beyond confirmation of the microarray results. For example, establishing an abnormal FISH signal pattern is a cost-effective approach for subsequent familial testing in the clinical setting. This familial testing is often necessary to determine whether a parent harbors the same damaging genomic imbalance identified in the proband. For example, up to $10 \%$ of probands who exhibit the well-characterized deletion of 22q11.2 inherit this genomic imbalance from a parent, who may be mildly affected..$^{11}$ Appropriate medical management of the parent and accurate recurrence risk calculations are dependent on assessment of the parents following the diagnosis of numerous microdeletion and microduplication syndromes, including 22q11.2 deletion syndrome, in the proband (Supplementary Figure S2 online).

One significant limitation of microarray is the inability to visualize the linear and chromosomal orientation of genetic material. In cases of large-scale genomic gains, adjunct metaphase FISH analysis can be used to visualize the precise chromosomal location of the extra genetic material identified by microarray. Duplications often are tandem on the chromosome of origin; however, in a subset of cases, the duplicated material resides on a different chromosome or in an atypical location on the chromosome of origin, which may occur as the result of an unbalanced translocation or an inversion. Characterization of these phenomena using FISH, at times in concert with conventional cytogenetics, is critical for an improved understanding of the biological consequences of the genomic imbalance and often for appropriate recurrence risk calculation.

A total of 86 cases from this study exhibited multiple genomic imbalances of reportable size. These imbalances are often indicative of structural rearrangements between chromosomes or within a single chromosome, resulting in derivative chromosome(s) (Supplementary Figure S3 online). In such cases characterization of the derivative chromosome(s) is critical because many are inherited from a parent with a balanced chromosome translocation. Identification of a balanced translocation in a parent significantly affects the risk of having future offspring with an 
Table 3 Recommended clinical strategy for adjunct testing for identified genomic imbalances following microarray

\begin{tabular}{|c|c|c|}
\hline Aberration type & Purpose of confirmation & Methodology \\
\hline Deletion $\geq 150 \mathrm{~kb}$ & Future familial studies & $\begin{array}{l}\text { FISH, MLPA, qPCR. Note: If the identified genomic imbalance is }>5- \\
10 \mathrm{Mb} \text { and is located in a defined banding area of the chromosome, } \\
\text { conventional cytogenetics may be considered. }\end{array}$ \\
\hline Duplication $\geq 500$ kb & Localization of aberration & $\begin{array}{l}\text { Metaphase FISH (concurrent interphase analysis recommended } \\
\text { to ensure detection of tandem duplication). Note: If the identified } \\
\text { genomic imbalance is }>10 \mathrm{Mb} \text { and is located in a defined banding area } \\
\text { of the chromosome, conventional cytogenetics may be considered. }\end{array}$ \\
\hline \multirow{2}{*}{$\begin{array}{l}\text { Multiple genomic imbalances } \\
\text { (deletions } \geq 150 \mathrm{~kb} \text { and/or } \\
\text { duplications } \geq 500 \mathrm{~kb} \text { ) }\end{array}$} & Future familial studies & See above for recommendations based on aberration type \\
\hline & Localization of aberration(s) & $\begin{array}{l}\text { Metaphase FISH (concurrent interphase analysis recommended to } \\
\text { ensure detection of tandem duplication) }\end{array}$ \\
\hline
\end{tabular}

FISH, fluorescence in situ hybridization; kb, kilobase; Mb, megabase; MLPA, multiplex ligation-dependent probe amplification; qPCR, quantitative polymerase chain reaction.

unbalanced chromosomal complement. Alternatively, identification of a similar genomic imbalance in a parent may offer insight into the clinical implications, if any, of the identified genomic imbalance. The methodology required to characterize the derivative chromosome(s) in patients with multiple genomic imbalances is dependent on the type and the location of the genomic imbalances. Conventional cytogenetics is extremely useful for characterization of large aberrations $(>10 \mathrm{Mb})$ and allows for assessment of the whole genome; however, resolution is dependent on the band level of the chromosomes being analyzed and the banding patterns of the target regions. Adjunct metaphase FISH analysis is often necessary to accurately characterize the orientation of genetic material in patients with complex findings (Supplementary Figure S3 online). The assay(s) used for subsequent parental studies should be informed by the proband's studies.

These data support a more limited use of adjunct testing solely for confirmatory purposes following microarray. A recommended clinical strategy for use of FISH and other orthogonal methodologies following the detection of largescale genomic imbalances (deletions $\geq 150 \mathrm{~kb}$ and duplications $\geq 500 \mathrm{~kb}$ ) by microarray is described in Table 3. Briefly, we recommend confirming isolated genomic imbalances (loss or gain) identified by microarray with a less expensive technology, such as FISH, when familial testing will be pursued. Adjunct testing is also recommended to determine the precise chromosomal localization of genomic gains and to characterize the chromosomal organization in patients with multiple genomic imbalances. The vast majority of current microarray platforms include both variable and nonvariable targets, allowing for internal verification of genomic imbalances. In a subset of cases, however, adjunct studies are necessary to clarify the microarray findings. To this end, we recommend that FISH or another technology capable of detecting genomic imbalances continue to be used when deemed technically necessary by the laboratorian (e.g., to resolve the chromosomal location of an identified genomic imbalance, particularly on the X chromosome).

It is important to note that this retrospective analysis evaluates the utility of confirmatory testing of large-scale genomic imbalances (deletions $\geq 150 \mathrm{~kb}$ and duplications $\geq 500 \mathrm{~kb}$ ) identified by microarray. These data do not allow for extrapolation to very small regions of imbalance identified with current high-resolution microarray platforms. The efficacy of confirmatory testing of small-scale imbalances warrants future assessment. In addition, this study highlights the importance of careful probe selection in cases of adjunct FISH analysis. Probe selection is influenced by the type (loss or gain), size, and precise location of the genomic imbalance being confirmed. Ideally, FISH probes used to confirm deletions should be contained within the known region of loss identified using microarray, restricting the practical utility of the FISH assay for deletions to $\sim 150 \mathrm{~kb}$. The FISH probe selected for confirmation of duplications should also be contained within the region of known gain. In addition, the amount of duplicated genetic material flanking the region covered by the FISH probe is important because a lack of sufficient distance between signals of a tandem or small inverted duplication during interphase analysis presents an interpretive challenge. In cases necessitating confirmation of genomic imbalances that are unsuitable for FISH analysis, alternative technologies, such as multiplex ligation-dependent probe amplification, quantitative polymerase chain reaction, or conventional cytogenetics, should be considered.

\section{SUPPLEMENTARY MATERIAL}

Supplementary material is linked to the online version of the paper at http://www.nature.com/gim 


\section{ACKNOWLEDGMENTS}

The authors thank Nicole Hackendahl for her assistance with manuscript preparation and Dianna Zaleski for her assistance with karyotype preparation.

\section{DISCLOSURE}

The authors declare no conflict of interest.

\section{REFERENCES}

1. Riegel M. Human molecular genetics: from cells to nucleotides. Genet Mol Biol 2014;37:194-209.

2. Manning M, Hudgins L; Professional Practice and Guidelines Committee. Array-based technology and recommendations for utilization in medical genetics practice for detection of chromosomal abnormalities. Genet Med 2010;12:742-745.

3. Miller DT, Adam MP, Aradhya S, et al. Consensus statement: chromosomal microarray is a first-tier clinical diagnostic test for individuals with developmental disabilities or congenital anomalies. Am J Hum Genet 2010;86:749-764.

4. Kang SH, Shaw C, Ou Z, et al. Insertional translocation detected using FISH confirmation of array-comparative genomic hybridization $(\mathrm{aCGH})$ results. $\mathrm{Am} \mathrm{J}$ Med Genet A 2010;152A:1111-1126.
5. Baldwin EL, Lee JY, Blake DM, et al. Enhanced detection of clinically relevant genomic imbalances using a targeted plus whole genome oligonucleotide microarray. Genet Med 2008;10:415-429.

6. Boone PM, Bacino CA, Shaw CA, et al. Detection of clinically relevant exonic copy-number changes by array CGH. Hum Mutat 2010;31:1326-1342.

7. Pickering DL, Eudy JD, Olney AH, et al. Array-based comparative genomic hybridization analysis of 1176 consecutive clinical genetics investigations. Genet Med 2008;10:262-266.

8. Kearney HM, Thorland EC, Brown KK, Quintero-Rivera F, South ST; Working Group of the American College of Medical Genetics Laboratory Quality Assurance Committee. American College of Medical Genetics standards and guidelines for interpretation and reporting of postnatal constitutional copy number variants. Genet Med 2011;13:680-685.

9. South ST, Lee C, Lamb AN, Higgins AW, Kearney HM; Working Group for the American College of Medical Genetics and Genomics Laboratory Quality Assurance Committee. ACMG Standards and Guidelines for constitutional cytogenomic microarray analysis, including postnatal and prenatal applications: revision 2013. Genet Med 2013;15:901-909.

10. Shaffer LG, McGowan-Jordan J, Schmid M, eds. ISCN (2013): An International System for Human Cytogenetic Nomenclature (2013). Karger: Basel, Switzerland, 2013.

11. McDonald-McGinn DM, Tonnesen MK, Laufer-Cahana A, et al. Phenotype of the 22 q11.2 deletion in individuals identified through an affected relative: cast a wide FISHing net! Genet Med 2001;3:23-29. 